\title{
Novel ECHS1 mutations in Leigh syndrome identified by whole-exome sequencing in five Chinese families: case report
}

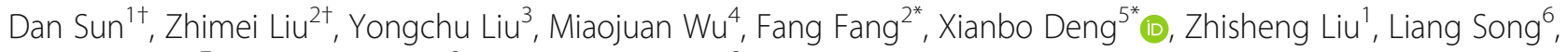
Kei Murayama', Chunhua Zhang ${ }^{8}$ and Yuanyuan Zhu $^{3}$

\begin{abstract}
Background: Short-chain enoyl-CoA hydratase deficiency (ECHS1D), also known as ECHS1 deficiency, is a rare inborn metabolic disorder with clinical presentations characterized by Leigh syndrome (LS). Thirty-four different pathogenic mutations have been identified from over 40 patients to date.

Case presentation: Here, we report five Chinese patients with clinical syndromes typified as LS. Despite different initial symptoms, all patients presented developmental regression, dystonia, common radiological features such as symmetrical bilateral brain abnormalities, and similar metabolic results such as elevated plasma lactate and 2,3dihydroxy-2-methylbutyrate. Utilizing whole-exome sequencing (WES), we identified eight distinct variants in ECHS1, with six novel variants, and the remaining two variants have been previously reported. Interestingly, one of the six novel variants, c.463G > A (p.Gly155Ser), was detected in three patients from unrelated families, suggesting a potential founder effect already described for a few mutations in LS. Incorporating both genetic analysis and medical results, including magnetic resonance imaging (MRI), electroencephalography (EEG), and biochemical testing, our study enriched the mutation spectrum of the ECHS1 gene and confirmed the phenotypic presentations of LS.

Conclusions: The severity of ECHS1 deficiency seems to vary. It was affected by both genetics and external environmental factors that lead to increased metabolism. Our study enriched the mutation spectrum of the ECHS1 gene, confirmed the phenotypic presentations, and highlighted the importance of the valine catabolic pathway in Leigh syndrome. Further studies are required to examine the potential founder mutation c.463G > A (p.Gly155Ser) and the role of ECHS1 in relevant pathways.
\end{abstract}

Keywords: Leigh syndrome, ECHS1, Whole-exome sequencing, Case report

\footnotetext{
*Correspondence: 13910150389@163.com; dengxianbo@hotmail.com

${ }^{\dagger}$ Dan Sun and Zhimei Liu contributed equally to this work.

2Department of Neurology, Beijing Children's Hospital, Capital Medical University, National Center for Children's Health, Beijing 100045, China

${ }^{5}$ Radiology Department, Union Hospital, Huazhong University of Science and Technology, Wuhan 430056, China

Full list of author information is available at the end of the article
}

(c) The Author(s). 2020 Open Access This article is licensed under a Creative Commons Attribution 4.0 International License, which permits use, sharing, adaptation, distribution and reproduction in any medium or format, as long as you give appropriate credit to the original author(s) and the source, provide a link to the Creative Commons licence, and indicate if changes were made. The images or other third party material in this article are included in the article's Creative Commons licence, unless indicated otherwise in a credit line to the material. If material is not included in the article's Creative Commons licence and your intended use is not permitted by statutory regulation or exceeds the permitted use, you will need to obtain permission directly from the copyright holder. To view a copy of this licence, visit http://creativecommons.org/licenses/by/4.0/ The Creative Commons Public Domain Dedication waiver (http://creativecommons.org/publicdomain/zero/1.0/) applies to the data made available in this article, unless otherwise stated in a credit line to the data. 


\section{Background}

Mitochondrial short-chain enoyl-CoA hydratase-1 deficiency, also known as ECHS1 deficiency, is an autosomal recessive inborn metabolic disorder commonly presenting after birth or early in life. It is typically characterized by developmental delay, regression, high levels of lactic acid, and abnormalities in the basal ganglia of the brain [1]. These clinical symptoms are consistent with Leigh syndrome (LS) or Leigh-like syndrome, which are rare heterogeneous progressive neurodegenerative disorders. In particular, $\mathrm{T}_{2}$ bilateral hyperintensities are characteristic of cranial magnetic resonance imaging (MRI) in LS [2, 3].

ECHS1 gene mutations were first discovered in two infant siblings in 2014 and reported to be one of the causes of LS [1]. These mutations are involved in the metabolic pathway of essential amino acids such as valine, as well as affecting the second step of mitochondrial fatty acid beta-oxidation (FAO). The reported clinical and biochemical characteristics of ECHS1 deficiency strongly indicated that the common pathological mechanism of ECHS1 deficiency is dysfunction of the valine catabolic pathway. The valine catabolic pathway has been elucidated to involve five enzymes. ECHS1 is responsible for the fourth step of valine degradation, which is to convert unsaturated trans-2-enoyl-CoA species, such as methacrylyl-CoA and acryloyl-CoA, to the corresponding 3(S)-hydroxyacyl-CoA [4]. The deficiency of ECHS1 leads to the accumulation of methacrylyl-CoA and acryloyl-CoA, two toxic intermediates that are highly reactive with sulfhydryl groups that are suspected to cause brain pathology and the relevant biochemical patterns [5].

Recently, Sharpe and McKenzie performed a comprehensive review of all reported cases of ECHS1 deficiency [2]. Briefly, thirty-four different mutations from 42 patients have been described to date. Most identified mutations are missense, and the majority of reported cases are compound heterozygous. Apart from c.476A > G (p.Gln159Arg), which has been found in multiple unrelated ECHS1-deficient patients of diverse ethnic origins [4, 6-9], no other hotspot mutations have been characterized.

Here, we report five patients from different Chinese families. They were diagnosed with LS with typical clinical symptoms such as developmental regression, dystonia, symmetrical bilateral abnormalities, and biochemical features such as increased 2,3-dihydroxy-2methylbutyrate and elevated plasma lactate, which strongly indicated ECHS1 deficiency. Whole-exome sequencing (WES) was performed on five patients to precisely identify the disease-causing genes and variants. Eight mutations in the ECHS1 gene were detected, six of which are novel. In particular, one of the novel mutations, c.463G > A (p.Gly155Ser), was detected in three of the five patients from unrelated families. Considering that the studied patients were all from the same region of China, it is a strong indication of a founder effect that has been reported in a few variants of ECHS1 deficiency in previous studies $[4,7,9]$. Our study enriched the mutation spectrum of the ECHS1 gene, confirmed the phenotypic presentations, and highlighted the importance of the valine catabolic pathway in Leigh syndrome.

\section{Case presentation Subjects}

Five patients from different families were diagnosed with LS in the Department of Pediatrics of Tongji Hospital in Wuhan and the Department of Neurology, Beijing Children's Hospital, Capital Medical University between 2016 and 2018. The study protocols were approved by ethics committees of both hospitals. Informed consent for routine and investigative genetic studies was obtained from parents of all studied patients and families.

\section{Metabolite analysis}

According to previous studies, urine 2,3-dihydroxy-2methylbutyrate, a special indicator on routine organic acid analysis, was measured for each studied patient [4, 10]. Acylcarnitine analysis of both butyl derivatives and plasma lactate/alanine/proline were also performed.

\section{Mitochondrial respiratory chain (MRC) enzyme activity measurement}

Two patients (patients 2 and 3) were arranged to measure enzyme activity by cultured skin fibroblasts. The mitochondrial marker enzyme citrate synthase (CS) and MRC complexes I, II, II + III, III, IV activity were detected in isolated mitochondria from skin fibroblasts, as described previously [11]. Enzyme activities of MRC complexes were calculated by the mean percentage of normal control relative to appropriate reference enzyme activities (such as CS). Enzyme activities in the cell line were defined as being decreased at $<40 \%$ [12].

\section{Oxygen consumption rate (OCR) measurement}

The OCR of fibroblasts was measured in two patients (patients 2 and 3) by an XF96 Extracellular Flux Analyzer (Seahorse Bioscience, Billerica, MA, USA). Samples were prepared according to descriptions in previous studies [13, 14]. Patient samples were measured together with two controls in each run. Patient fibroblast cell lines and controls were seeded in at least 14 wells of two XF96 cell culture microplates (Seahorse Bioscience) at a cell density of 20,000 per $80 \mu \mathrm{L}$ of growth medium in each well and incubated overnight $\left(5 \% \mathrm{CO}_{2}, 37^{\circ} \mathrm{C}\right)$. The growth medium was then replaced with $160 \mu \mathrm{l}$ of 
$25 \mathrm{mM}$ glucose medium or $10 \mathrm{mM}$ galactose medium the following day. The microplate was placed into a $\mathrm{CO}_{2}$-free incubator at $37^{\circ} \mathrm{C}$ for $60 \mathrm{~min}$ before measurement. After the measurement of basal OCR, $10 \mu \mathrm{M}$ oligomycin, $4 \mu \mathrm{M}$ carbonyl cyanide phenylhydrazone (FCCP), and $20 \mu \mathrm{M}$ rotenone were added, followed by OCR recording upon each addition [13]. The maximum respiration rate (MRR) corresponds to the OCR after FCCP injection minus rotenone-insensitive OCR. MRR was denoted as a percentage relative to the average of the controls. A reduction of less than $71.6 \%$ was considered a significant decline [14].

\section{Genetic analysis}

Due to the genetic heterogeneity where LS can be caused by genetic abnormalities from more than 75 genes, WES and analysis for the detection of variants were performed on all five patients. Briefly, genomic DNA was extracted from whole blood samples. Whole exons and flanking intronic sequences were captured by an Agilent Sure-Select Human All Exon Kit v6, followed by high-throughput sequencing on the Illumina HiSeq 2000 platform with 150-bp paired-end reads. Bioinformatic analysis was carried out with public software and a self-developed pipeline. Specifically, all cleaned data after trimming were aligned against the human reference genome build hg19 using BWA [15]. The average depths of WES data ranged from $95 \mathrm{x}$ to $171 \mathrm{x}$ across five patients.

Single nucleotide variants (SNVs) and indels were then discovered by HaplotypeCaller of GATK, followed by variant annotation through ANNOVAR [16], integrating customized databases such as ClinPred [17]. The analysis was restricted to coding and flanking intronic regions $( \pm$ $20 \mathrm{bp}$ ) where good data coverage is normally guaranteed. Variants with minor allele frequency (MAF) greater than $1 \%$ in any of the gnomAD, ExAC, 1000 Genomes Project, and ESP6500 databases were excluded from subsequent analysis. Filtering and prioritizing were then performed to look into potential detrimental variants such as nonsense, missense, frameshift, and variants impacting splice for variant interpretation and classification according to American College of Medical Genetics (ACMG) guidelines [18].

To confirm the mutations identified by WES, fragments covering the mutation site in ECHS1 were amplified by PCR followed by Sanger sequencing. PCR was conducted with Premix Taq ${ }^{\text {ma }}$ Hot Start Version/TaKaRa LA Taq ${ }^{\circledR}$ with GC Buffer (Takara, Osaka, Japan) following the following protocol: $95^{\circ} \mathrm{C}$ for $2 \mathrm{~min}$, then 35 cycles consisting of $95^{\circ} \mathrm{C}$ for $30 \mathrm{~s}, 60^{\circ} \mathrm{C}$ for $30 \mathrm{~s}$ and $72{ }^{\circ} \mathrm{C}$ for $30 \mathrm{~s}, 72^{\circ} \mathrm{C}$ for $5 \mathrm{~min}$ in the end. PCR products were purified and sequenced on the ABI 3730 DNA
Analyzer using the BigDye ${ }^{\mathrm{Tm}}$ Terminator Cycle Sequencing Kit (Applied Biosystems, Foster, CA, USA).

\section{Results \\ Clinical features, brain MRI examinations, and biochemical investigations}

The studied patients were from five different and unrelated Chinese families with healthy and nonconsanguineous parents. Their ages of onset ranged from birth to 21 months, with diverse initial symptoms. However, they presented similar clinical features, MRI findings, and biochemical results, such as developmental regression; bilateral abnormal signals, especially around basal ganglia; and elevated serum lactate, respectively. These characteristics strongly indicated that they have the same disease or syndrome. Table 1 summarizes the general information, clinical features, brain MRI findings, and biochemical examination results of all patients. We describe each patient in detail in the following section.

Patient 1 is currently 4 years and 7 months old. He was born by caesarean section at full term and exhibited normal developmental milestones until initial presentations of nystagmus at 6 months. Arrested development of being unable to sit steadily started to be noticed at the age of 8 months. He underwent the first brain MRI examination at 8 months and an enlarged subarachnoid space and hyperintensities of the right caudate head and bilateral globus pallidus were found in T2-weighted images. He then received rehabilitation training but without visible improvement. Global regression with gradually progressive extrapyramidal dyskinesia and dystonia was noted at the age of 10 months. His second brain MRI was carried out at 12 months because other symptoms started to appear. Bilateral lesions of the cerebral peduncle and basal ganglia, including the globus pallidus, caudate nucleus, and putamen, as well as brain atrophy, were observed.

His initial biochemical investigations showed marginally elevated lactate and pyruvate in plasma. The lactate/ pyruvate ratio was measured, and the value was 13.3. However, no significant abnormality was found in the acylcarnitine analysis. Urine organic acid analysis also showed only a slightly abnormal increase in 2,3-dihydroxy-2-methylbutyrate.

Patient 2 was the only female child among the five patients. She is the first child of a family with a healthy half-sister from the same father. Her first manifestation was a sudden convulsion that lasted for $90 \mathrm{~min}$ at the age of 21 months. Development milestones were reported by parents to be normal previously. Blood gas analysis was carried out to show metabolic acidosis. She had her first brain MRI at admission and showed bilateral basal ganglia lesions that mainly involved the globus pallidus, where the right side was more severe than the 
Table 1 Summary of general information, clinical features, brain magnetic resonance imaging and biochemical examinations of five patients

\begin{tabular}{|c|c|c|c|c|c|}
\hline & Patient 1 & Patient 2 & Patient 3 & Patient 4 & Patient 5 \\
\hline \multicolumn{6}{|c|}{ General information and clinical features } \\
\hline Gender & Male & Female & Male & Male & Male \\
\hline Age of onset & 6 months & 21 months & 17 months & At birth & 10 months \\
\hline Current age & 4 years and 7 months & 6 years & 29 months & 8 months & 19 months \\
\hline $\begin{array}{l}\text { Initial } \\
\text { presentation }\end{array}$ & Nystagmus & regression & Episodes of dystonia & Paroxysmal dyskinesia & $\begin{array}{l}\text { Diarrhea, eyes on the } \\
\text { turn, myotonia of the } \\
\text { lower limb }\end{array}$ \\
\hline $\begin{array}{l}\text { Central Nervous } \\
\text { System }\end{array}$ & $\begin{array}{l}\text { Global developmental } \\
\text { delay (HP:0001263); } \\
\text { Developmental } \\
\text { regression (HP:0002376); } \\
\text { Nystagmus (HP: } \\
\text { 0000639); } \\
\text { Extrapyramidal } \\
\text { dyskinesia (HP:0007308); } \\
\text { Babinski sign (HP: } \\
\text { 0003487); } \\
\text { Dystonia (HP:0001332) }\end{array}$ & $\begin{array}{l}\text { Developmental regression } \\
\text { (HP:0002376); } \\
\text { Dysarthria (HP:0001260); } \\
\text { Dystonia (HP:0001332) }\end{array}$ & Dystonia (HP:0001332) & $\begin{array}{l}\text { Motor deterioration (HP: } \\
\text { 0002333) }\end{array}$ & $\begin{array}{l}\text { Developmental } \\
\text { regression (HP:0002376) }\end{array}$ \\
\hline \multicolumn{6}{|c|}{ Brain magnetic resonance imaging } \\
\hline $\begin{array}{l}\text { Globus } \\
\text { pallidus }\end{array}$ & + & + & + & + & + \\
\hline Putamen & + & + & - & + & + \\
\hline $\begin{array}{l}\text { Caudate } \\
\text { nucleus }\end{array}$ & + & + & - & + & + \\
\hline Brain stem & + & - & - & + & - \\
\hline Summary & $\begin{array}{l}\text { Symmetric lesions of } \\
\text { the basal ganglia (HP: } \\
\text { 0007039); } \\
\text { Brain atrophy (HP: } \\
\text { 0012444) }\end{array}$ & $\begin{array}{l}\text { Symmetric lesions of the } \\
\text { basal ganglia (HP:0007039) }\end{array}$ & $\begin{array}{l}\text { Symmetric lesions of } \\
\text { the basal ganglia (HP: } \\
\text { 0007039) }\end{array}$ & $\begin{array}{l}\text { Symmetric lesions of } \\
\text { the basal ganglia (HP: } \\
\text { 0007039) }\end{array}$ & $\begin{array}{l}\text { Symmetric lesions of the } \\
\text { basal ganglia (HP: } \\
\text { 0007039) }\end{array}$ \\
\hline \multicolumn{6}{|c|}{ Biochemical examination } \\
\hline $\begin{array}{l}\text { Plasma } \\
\text { lactate } \\
\text { (mmol/L) }\end{array}$ & $\begin{array}{l}1.6->3.4 \\
(0.5-2.2)\end{array}$ & $\begin{array}{l}1.68 \\
(0.5-2.2)\end{array}$ & $\begin{array}{l}3.17->6.46 \\
(0.5-2.2)\end{array}$ & $\begin{array}{l}11.41 \\
(0.5-2.2)\end{array}$ & $\begin{array}{l}2.51 \\
(0.5-2.2)\end{array}$ \\
\hline $\begin{array}{l}\text { Plasma } \\
\text { pyruvate } \\
\text { (umol/L) }\end{array}$ & $197-255$ & Not performed & Not performed & Not performed & Not performed \\
\hline $\begin{array}{l}\text { Acylcarnitine } \\
\text { analysis } \\
\text { (DBS) }\end{array}$ & Normal profile & Normal profile & Normal profile & Slight increase in $\mathrm{C} 4 \mathrm{OH}$ & Normal profile \\
\hline $\begin{array}{l}\text { Organic acid } \\
\text { analysis }\end{array}$ & $\begin{array}{l}\text { Increases in } \\
\text { 2,3-dihydroxy-2- } \\
\text { Methylbutyrate }(0.0446)\end{array}$ & $\begin{array}{l}\text { Increases in 3- } \\
\text { hydroxyisovaleric acid and } \\
\text { 2,3-dihydroxy-2- } \\
\text { Methylbutyrate }(0.0445)\end{array}$ & $\begin{array}{l}\text { Increases in } \\
\text { 2,3-dihydroxy-2- } \\
\text { Methylbutyrate }\end{array}$ & $\begin{array}{l}\text { Increases in } \\
\text { 2,3-dihydroxy-2- } \\
\text { Methylbutyrate }\end{array}$ & $\begin{array}{l}\text { Increases in } \\
\text { pyruvate }\end{array}$ \\
\hline Skin biopsy & Not performed & $\begin{array}{l}\text { OCR:79\%/78\% (Glucose/ } \\
\text { Galactose medium) } \\
\text { MRC enzyme activity } \\
\text { measurement: no } \\
\text { significance }\end{array}$ & $\begin{array}{l}\text { OCR:56\%/71\% (Glucose/ } \\
\text { Galactose medium) } \\
\text { MRC enzyme activity } \\
\text { measurement: no } \\
\text { significance }\end{array}$ & Not performed & Not performed \\
\hline
\end{tabular}

left. No epileptic discharge was found in the EEG results. Global developmental regressions, such as motor and language, were manifested thereafter. She then received rehabilitation training, with visible but limited benefits, showing that she could walk unsteadily at 3 years old, as well as be capable of learning and understanding simple instructions but without active communication. She underwent brain MRI reexamination at the age of 4 years and 7 months. Bilateral lesions of the globus pallidus, caudate nucleus, and putamen were evident; 
however, the lesions of the globus pallidus were smaller than the previous examination.

The biochemical analysis showed that her plasma lactate was normal. For urine organic acid testing, 3hydroxyisovaleric acid and 2,3-dihydroxy-2-methylbutyrate were abnormally increased. Acylcarnitine was at a normal level. Skin biopsy demonstrated normal OCR (79\%/78\%) and enzyme activity of the respiratory chain.

Patient 3 is also the first child in his family, with almost normal development milestones. At the age of 17 months, he started to manifest dystonia with an unexplained cause. The dystonia normally lasted for one minute but could be up to $30 \mathrm{~min}$ in severe cases. He appeared normal in between two dystonia phases. In addition, salivation and choking were ordinarily observed during water drinking. His biomedical tests showed metabolic acidosis and elevated serum lactate but no specific changes in organic acid and amino acid profiles. He also underwent two brain MRI examinations at the ages of 17 months and 21 months, presenting similar results with bilateral globus pallidus involvement.

His plasma lactate showed mild elevation. The level of 2,3-dihydroxy-2-methylbutyrate was increased; however, acylcarnitine was normal. Skin biopsy showed that OCR was abnormally decreased (56\%/71\%) but with essentially normal enzyme activity of the respiratory chain.

Patient 4 was admitted to the hospital at 8 months old, the youngest child of the five patients. He is the second child in his family with a healthy brother and was delivered vaginally with paroxysmal dyskinesia at his birth. He was unable to raise his head at the moment of admission. He underwent a brain MRI examination twice. The results of the first study showed abnormal symmetrical bilateral signals in the basal ganglia, thalamus, and midbrain cerebral peduncle, as well as an enlarged subarachnoid space (see Fig. 1). The second examination was performed 17 days later and demonstrated that both brain stem and basal ganglia present abnormal bilateral signals.

In the biochemical analysis, plasma lactate and hydroxybutyrylcarnitine $\left(\mathrm{C}_{4} \mathrm{OH}\right)$ were elevated. In addition, 2, 3-dihydroxy-2-methylbutyrate was increased based on the urine organic acid analysis. When he returned for review, at the age of 13 months, he was still unable to raise his head, sit steadily or speak. The level of serum lactate increased slightly.

Patient 5 was the younger of identical twins. He was admitted to our hospital at the age of 10 months with (a1)

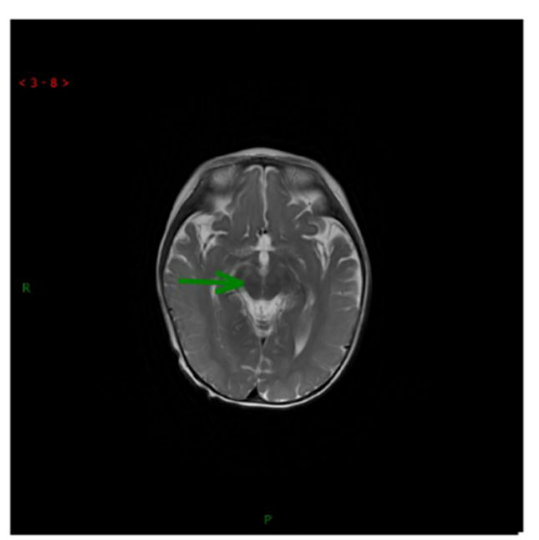

(b1)

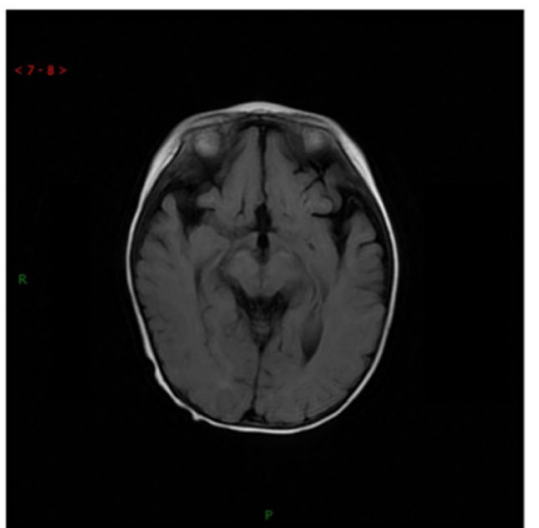

(a2)

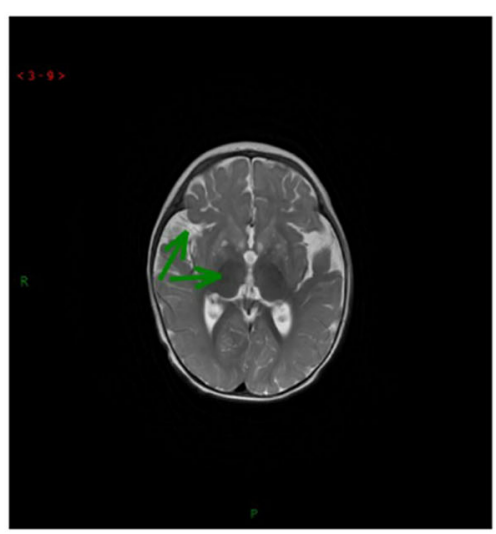

(b2)

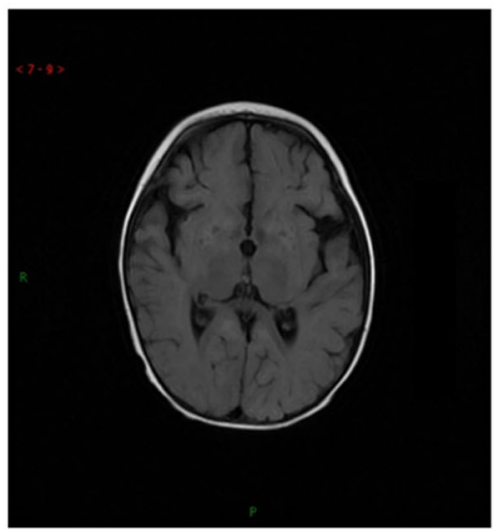

Fig. 1 MRI and MRS of patient P4. Images (a1) and (a2) are T2-weighted images, with green arrows showing abnormal areas. (b1) and (b2) are T2-FLAIR images 
diarrhoea and myotonia of the lower limb, as well as developmental regression including intellectual, motor, speech, and language. A slightly elevated slow wave was found in the EEG. Brain MRI examination showed an asymmetric abnormal signal of the bilateral basal ganglia (see Fig. 2). Urine organic acid analysis revealed increased pyruvate. He still had difficulty controlling his head and body after 12 days of treatment. He then started rehabilitation training at the age of 15 months. During this period, he underwent the second MRI and EEG examinations, which still showed marked bilateral partial basal ganglia and massive profound fast waves, respectively. At 16 months, he was admitted to our hospital again with vomiting. His physical examination reported that he had appendicular motor hypertonia, mild genu valgum, and calcaneovalgus deformity, as well as being incapable of sitting steadily. The biochemical test showed an increased level of pyruvate in urine organic acid analysis and a slight elevation in plasma lactate levels but normal acylcarnitine levels. His twin brother presented syndromes similar to him but mildly.

\section{Genetic analysis \\ Family 1}

One of the identified biallelic mutations of patient 1 , c. $5 \mathrm{C}>\mathrm{T}$ (p.Ala2Val), in exon 1 of the ECHS1 gene (NM_004092.3) has been previously reported to be likely pathogenic, with functional verification $[19,20]$. The other mutation, c.607C $>\mathrm{T}$ (p.Ala203Thr), which is located in exon 5, is novel. Multiple lines of computer programs predict that the mutation is damaging. The population allele frequencies of both mutations are either much lower than $1 \%$ in or not even present in the population frequency databases (Table 2). We searched our in-house database, which consists of approximately 5000 samples, and found no samples with either mutation. Sanger sequencing was performed on the patients and their parents and confirmed that c.5G > A (p.Ala2Val) and c.607C $>\mathrm{T}$ (p.Ala203Thr) are inherited from his heterozygous mother and father, respectively (see Fig. 3).

\section{Family 2}

Biallelic mutations, c.463G $>$ A (p.Gly155Ser) and c.557C > T (p.Ser186Leu), were identified in patient 2 . Neither has been previously reported. Similar to patient 1 , the population allele frequencies of both mutations were much lower than $1 \%$ in all databases (Table 2). Multiple computer programs predicted that the two mutations are damaging. Sanger sequencing confirmed that her parents are both heterozygous for the ECHS1 gene carrying variants c.463G > A (p.Gly155Ser) and c.557C > $\mathrm{T}$ (p.Ser186Leu), respectively.

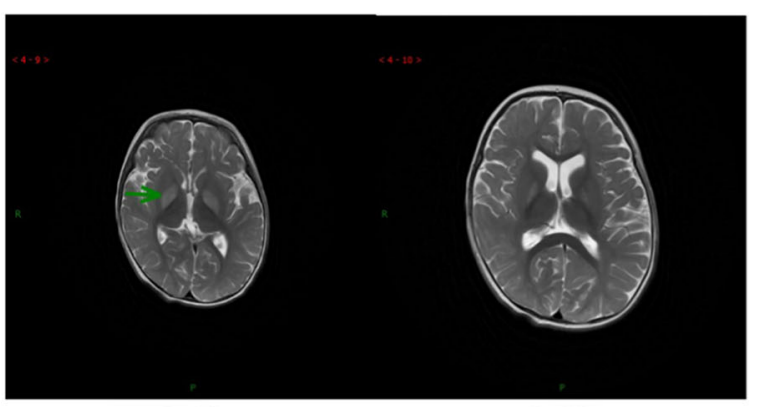

(a1)

(a2)

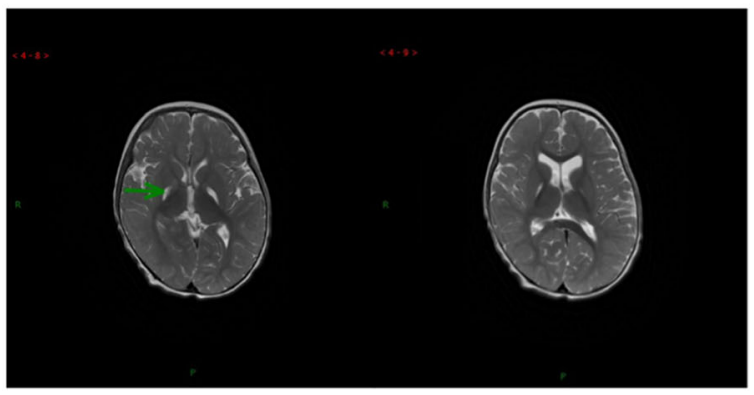

(a3)

(a4)

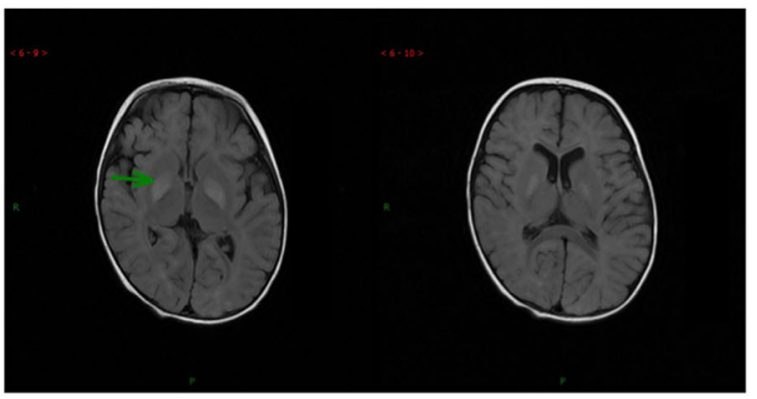

(b1)

(b2)

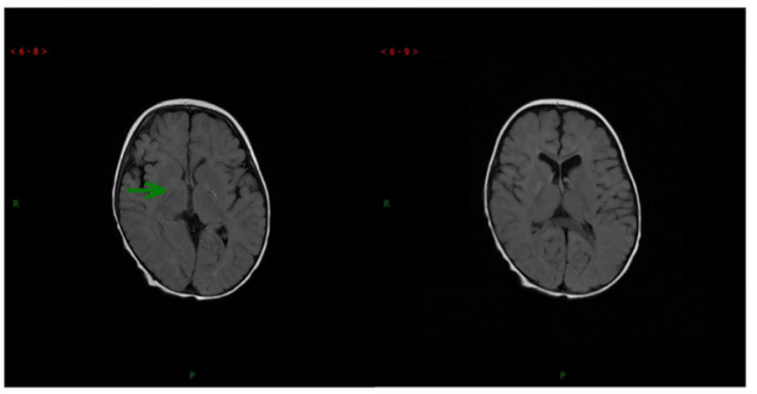

(b3)

(b4)

Fig. 2 MRI images of patient P5 with green arrows showing abnormal areas. Images (a1-4) and (b1-4) are T2-weighted and T2FLAIR images, respectively. Images (a $1-2)$ and (b1-2) are the results of the first MRI, which show symmetric abnormal signals of the bilateral basal ganglia. Images (a3-4) and (b3-4) show the results of the second MRI, which were similar to the first MRI

\section{Family 3}

For patient 3, the identified variants were c.583G >A (p.Gly195Ser) and c.463G > A (p.Gly155Ser), which were 
Sun et al. BMC Medical Genetics ～～(2020) 21:149

Page 7 of 12

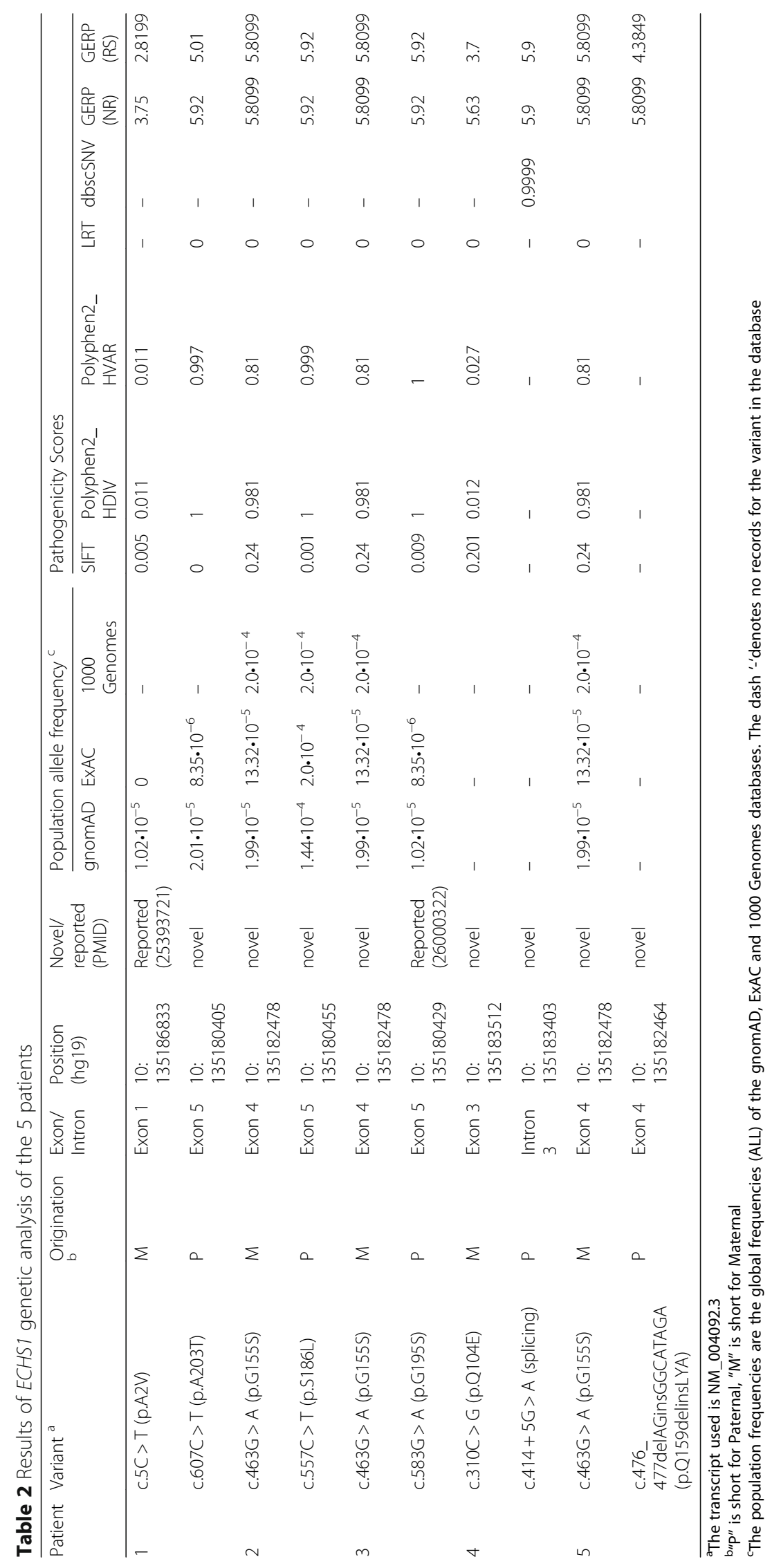




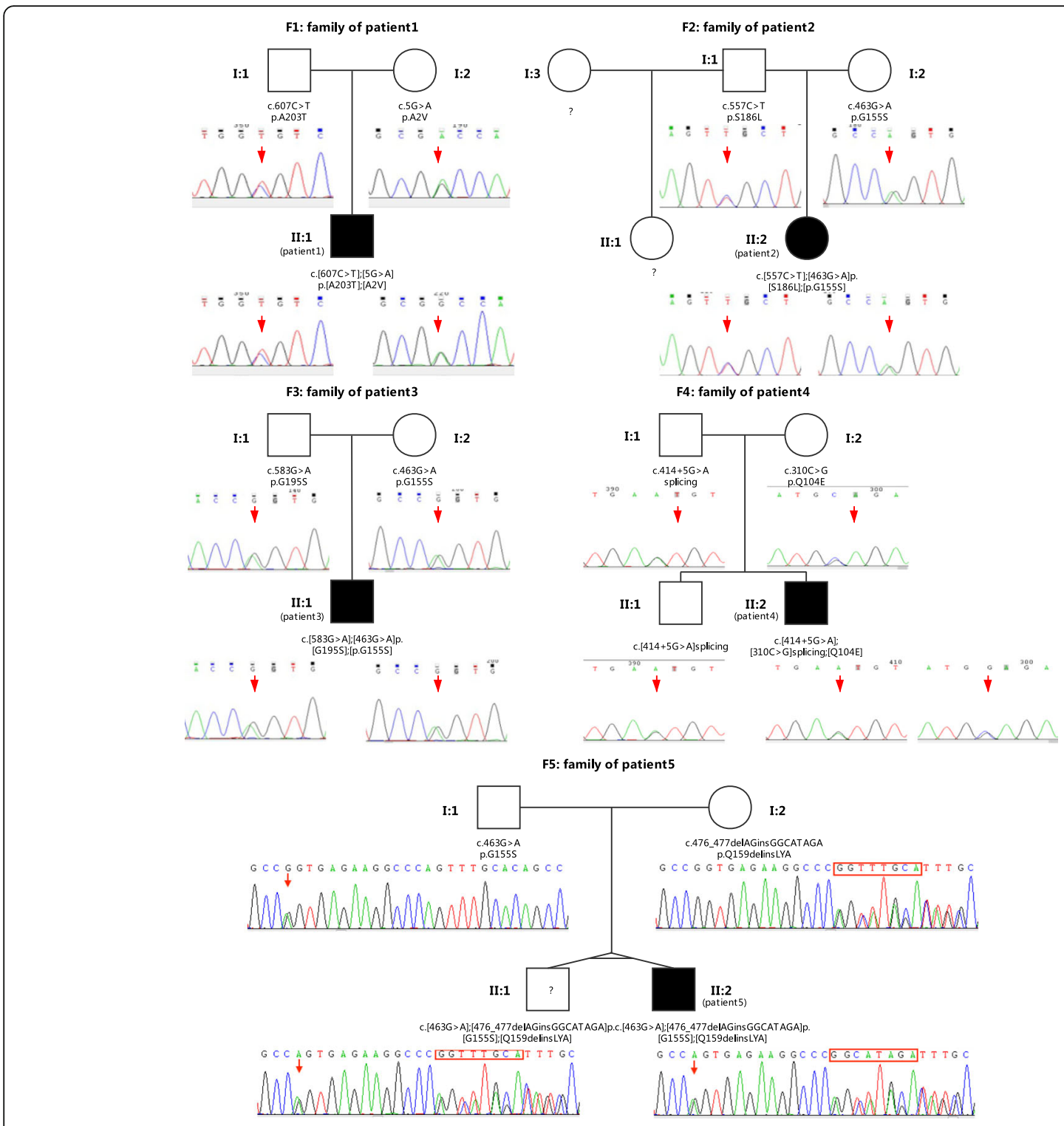

Fig. 3 Genograms with Sanger confirmation results for the five studied families

in exon 5 and exon 4 of $E C H S 1$, respectively. The first has been previously reported [7], with a population allele frequency lower than $1 \%$ for the global population in gnomAD. The second is the same as one of the identified mutations of patient 2. Multiple computer programs predicted that the two mutations are damaging. The patient's father was confirmed to be heterozygous for c.583G > A in ECHS1, and his mother was confirmed to be heterozygous for c.463G > A.

\section{Family 4}

Unlike the mutations identified for the previous three patients, the intron mutation c. $414+5 \mathrm{G}>\mathrm{A}$ in intron 3 of the ECHS1 gene was identified in patient 4 . The computer programs (GERP, dbscSNV) predict that the site is conserved and that the mutation most likely leads to splicing alteration. The other variant identified is c.310C > G (p.Gln104Glu) in exon 3. Both are novel mutations that have never been reported in the literature or recorded in databases. Sanger sequencing confirmed that the variants were inherited from his father (c.414+ $5 \mathrm{G}>\mathrm{A})$ and mother $(\mathrm{c} .310 \mathrm{C}>\mathrm{G})$. Both parents are heterozygous for the ECHS1 gene.

\section{Family 5}

An indel variant in exon 4 of ECHS1, c.476_477delAGinsGGCATAGA, which was inherited from his father, was identified. It has never been mentioned in previous studies, interpretations, or any databases. However, it is worth noting that c.476A > G (p.Gln159Arg) is a hotspot 
mutation for ECHS1D in ClinVar and was categorized as "pathogenic/likely pathogenic". Taking both facts together into consideration, this variant can be regarded as a combination of two isolated mutations of c.476A $>\mathrm{G}$ (p.Gln159Arg) and c.478insCATAGA. Hence, it is rational to consider that this allele has a likely pathogenic variant. The other variant confirmed to originate from the patient's mother is c.463G > A (p.Gly155Ser), which was also present in patients 2 and 3 .

In summary, the five patients in our study were all compound heterozygous, with different variants in ECHS1 alleles inherited from their parents. Sanger sequencing was carried out for each family to verify the variant inheritance (Fig. 3). The results of the genetic analysis are summarized in Table 2.

\section{Discussion and conclusions}

In this study, we reported five patients presenting diverse initial symptoms but consistent later clinical features with LS. Regarding genetic heterogeneity, wholeexome sequencing was performed to uncover diseasecausing genes and mutations.

During the genetic analysis, none of the five patients were found to have mutations in any previously reported nuclear genes associated with LS except for ECHS1. Mitochondrial DNA sequencing was also performed for patients 2 and 3 without any explainable mutations identified. Finally, we identified eight distinct mutations; six were novel, and the other two have been previously reported [7, 19]. As shown in Table 2, all detected mutations are extremely rare in public population databases, with a few of them not even present. In addition, none of these mutations were found in our in-house database with approximately 5000 samples. Each of these mutations is classified as pathogenic or detrimental by at least one or more prediction methods.

Sharpe and McKenzie performed a comprehensive review of ECHS1D and collected all reported ECHS1 mutations up to then in 2018 [2]. Carlston and colleagues updated the reported mutations in their case report of ECHS1D [21]. To date, 34 pathogenic ECHS1 mutations have been identified, with 29 missense, 2 splicing, 2 frameshift and 1 nonsense [2, 21]. We summarized the currently reported mutation spectrum of the ECHS1 gene in Fig. 4. The same mutational pattern was also observed in our study, where the major mutation type is missense. Specifically, six of the eight identified mutations in our study are missense, with one splicing and one indel. It is worth noting that one of the novel mutations, c.463G > A (p.Gly155Ser), was detected in three of the five studied patients. ClinVar interpretation of pathogenicity is "uncertain significance". However, combining multiple supporting lines of evidence, such as extremely low population allelic frequency, located in a functional domain without benign variations, multiple computational supporting evidence of being deleterious, as well as being a missense mutation, which is the common mechanism of ECHS1D, together with the fact that it was detected in three unrelated families in our study, we strongly suggested that it be recategorized as pathogenic or likely pathogenic. Moreover, this is also an indication of a founder effect that requires indepth research and has been mentioned in a few ECHS1D studies [4, 7].

Clinically, despite different initial presentations, all studied patients presented similar clinical syndromes, such as development regression, paroxysmal exerciseinduced dystonia, and common radiological features, such as symmetrical bilateral abnormalities. Moreover, the plasma lactate of most patients was elevated, together with a normal acylcarnitine profile and elevated erythro-2,3-dihydroxy-2-methylbutyrate levels. All these symptoms are consistent with other studies reporting Leigh syndrome [2, 4, 22, 23].

ECHS1 is a mitochondrial matrix enzyme that catalyses multiple metabolic pathways, such as fatty acids and valine oxidation. Recent studies reported that ECHS1 mutations are the causes of severe early-onset Leigh-like mitochondrial encephalopathy, accompanied by deafness, epileptic seizures, and optic atrophy, as well as feeding problems and cardiomyopathy $[1,2,4]$. To date, we still do not know this mechanism. According to the consistently elevated lactate level, it is speculated that the accumulation of harmful intermediate metabolites may lead to brain toxicity and disorders of mitochondrial energy metabolism $[1,2]$. In some cases, the marked presence of metabolite 2-methyl-2,3-hydroxybutyric acid in urine organic acids has been detected. The metabolic pathway of 2-methyl-2,3-dihydroxybutyrate is currently unclear and needs more in-depth research in the field.

The severity of ECHS1 deficiency seems to vary. It is affected by both genetics and external environmental factors that lead to increased metabolism [7]. Among our patients, three showed a sharp increase in the severity of symptoms after acute viral infections. This may be due to the elevated energy consumption in the decomposition states. Compared with early reported lethal cases [24], the results of biochemical testing can be complex and diverse, and metabolic abnormalities are often nonspecific.

Elevated pyruvate and lactate accompanied by normal pyruvate-lactate ratios have been observed in a few patients with early-onset lactic acidosis. Due to the heterogeneity of the natural course of ECHS1D, metabolic abnormalities at any step in the pathway may lead to changes in metabolic demands [21]. 
A ECHS1 variants

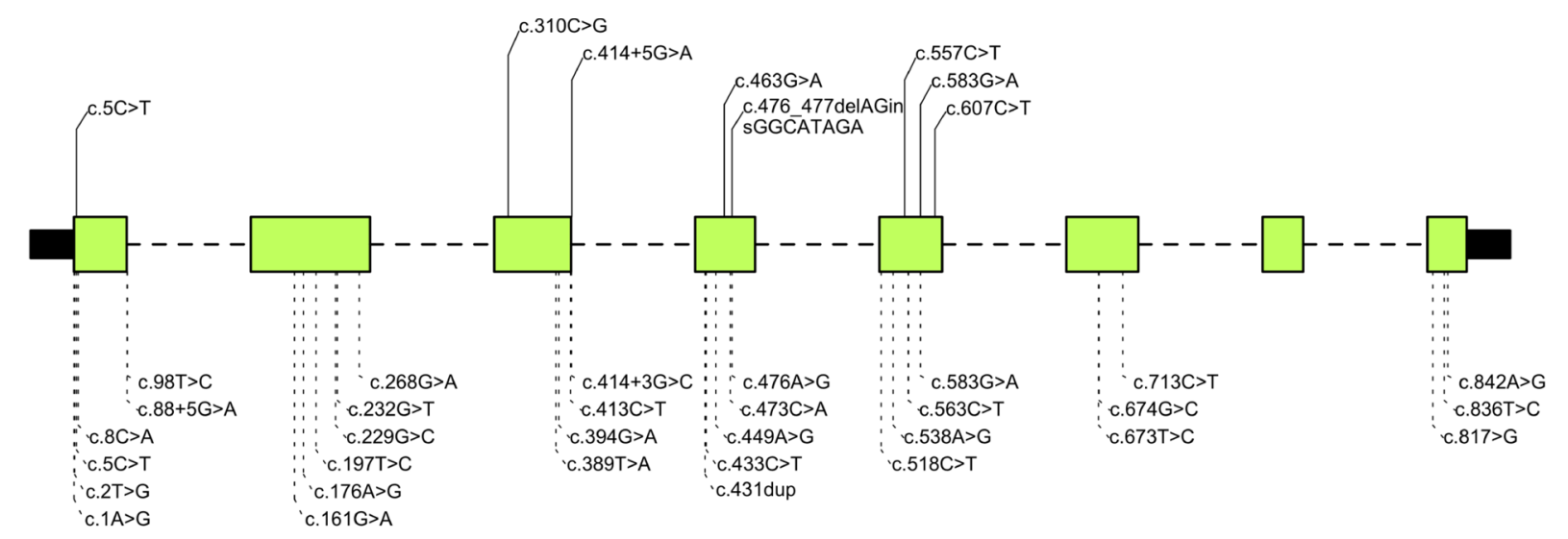

B ECHS1 amino acid alterations

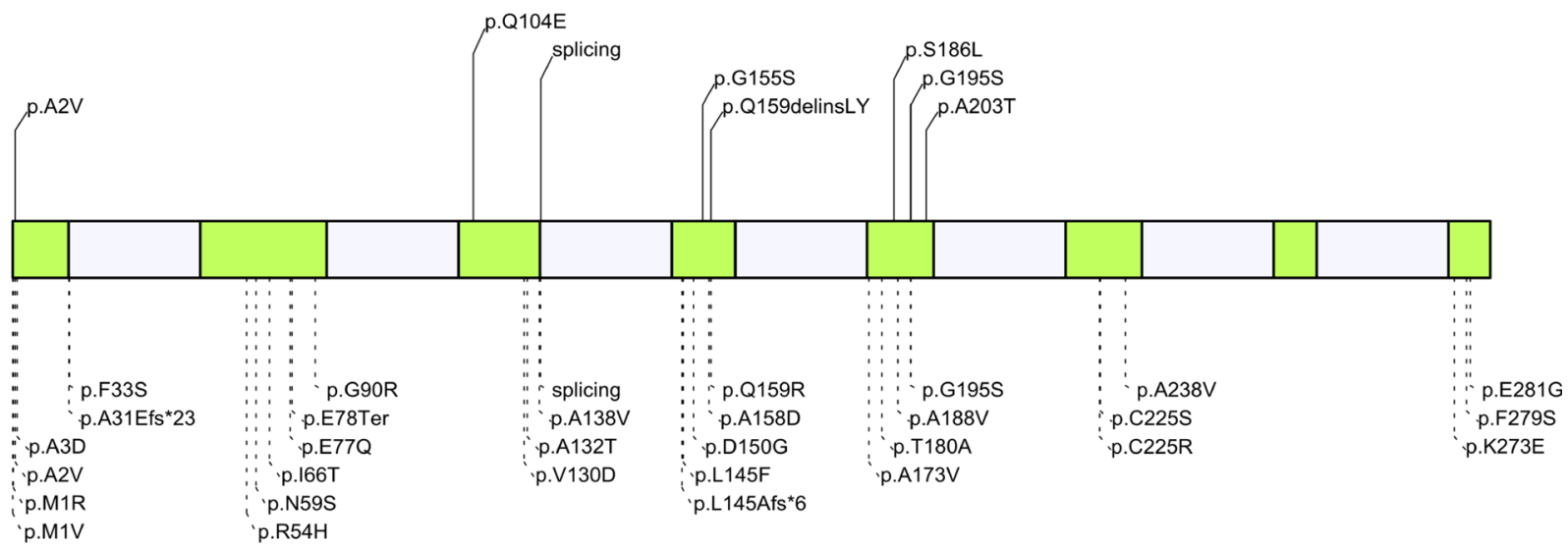

Fig. 4 Summary of the variants and amino acid alterations of ECHS1 gene related to Leigh syndrome. (A) The previously reported variants (below the bar with dashed lines) and the newly identified variants (above the bar with solid lines); (B) The previously reported amino acid alterations (below the bar with dashed lines) and the newly identified amino acid alterations (above the bar with solid lines)

Mutations in the $H I B C H$ gene have recently also been described as being the cause of Leigh-like syndrome. Abnormalities in metabolites indicate that $\mathrm{HIBCH}$ deficiency affects valine metabolism. The clinical symptoms of $\mathrm{HIBCH}$ deficiency were developmental delay, regression after an acute viral infection, dystonia, and bilateral basal ganglia abnormalities observed in MRI brain images. In the valine catabolic pathway, $\mathrm{HIBCH}$ is involved in the preceding step of ECHS1. The defect of HIBCH leads to metabolic pathway blockade and metabolite (substrate and methacrylyl-CoA) accumulation. Excess methacrylylCoA may react with thiol compounds, while cysteine residues, which are essential to mitochondrial enzymes, translate to thiol conjugates of methacrylylCoA. This series of changes caused a reduction in the cellular reduction state and ATP production, resulting in nerve cells in the basal ganglia [24]. ECHS1 and $\mathrm{HIBCH}$ deficiencies have many similarities in clinical and biochemical characteristics. Both can manifest feeding difficulties from the neonatal period, psychomotor retardation, dystonia, lactic acidosis, bilateral basal ganglia signal abnormalities on brain MRI, and sharp aggravation of the condition after acute viral infection [7].

The first time ECHS1 mutations were identified as one of the causes of LS was in 2014, thanks to the rapid progress of the clinical application of next-generation sequencing technology. To date, more than thirty mutations from over 40 families have already been discovered. Here, we followed the same strategy as most previous works, implementing WES for the discovery of mutations and Sanger sequencing for mutation verification. Our findings enriched both the current mutation spectrum of the ECHS1 gene and the phenotypic presentations of ECHS1D, especially in the Chinese population, as well as highlighting the importance of the valine catabolic pathway in Leigh syndrome. 


\section{Abbreviations}

ECHS1D: Short-chain enoyl-CoA hydratase deficiency; LS: Leigh syndrome; WES: Whole-exome sequencing; MRI: Magnetic resonance imaging; EEG: Electroencephalography; FAO: Fatty acid beta-oxidation; MRC: Mitochondrial respiratory chain; CS: Citrate synthase; OCR: Oxygen consumption rate; FCCP: Carbonyl cyanide phenylhydrazone; MRR: Maximum respiration rate; MAF: Minor allele frequency

\section{Acknowledgements}

Not applicable.

\section{Authors' contributions}

$F F$, XBD, and ZSL were responsible for the design of the study. DS, ZML, MJW, and LS were responsible for the clinical examination of the patients, data collection, and interpretation of the data from the clinical, imaging, and laboratory tests. DS drafted the manuscript. $\mathrm{KM}$ and $\mathrm{CHZ}$ were responsible for the interpretation of gene mutations detected in the study and revised the manuscript. YCL and YYZ were responsible for the analysis and interpretation of sequencing data. The authors have read and approved the final manuscript.

\section{Funding}

The study was supported by grants from the National Key Research and Development Program of China (No. 2016YFC1306202) and the Futang Children's Science Foundation Project (FTCSF-2018-5). The cost of sequencing was borne by the two funding projects.

\section{Availability of data and materials}

The datasets analysed during the current study are available in the NCBI Sequence Read Archive (SRA) repository [SRA accession: PRJNA637796, https://www.ncbi.nlm.nih.gov/sra/PRJNA637796]. The humanG1Kv37 (human_g1k_v37.fasta, MD5sum: 0ce84c872fc0072a885926823dcd0338) reference is equivalent to b37, with the exception that it does not contain the decoy sequence for human herpesvirus 4 type 1 (named NC007605 ). This reference grew out of the 1000 Genomes Project. http://ftp.1 000genomes.ebi.ac.uk/vol1/ftp/technical/reference/human_g1k_v37.fasta.gz.

\section{Ethics approval and consent to participate}

This research was approved by the Medical Ethics Committee of Wuhan Children's Hospital (Wuhan Maternal and Child Healthcare Hospital) and the Medical Ethics Committee of Beijing Children's Hospital, Capital Medical University. Written informed consent was obtained from the patient's parents for the participation of this study.

\section{Consent for publication}

The patient's parents provided written consent for the case report to be published.

\section{Competing interests}

The authors declare that they have no competing interests.

\section{Author details}

'Department of Pediatric Neurology, Wuhan Children's Hospital, Tongji Medical College, Huazhong University of Science \& and Technology, Wuhan 430016, China. 'Department of Neurology, Beijing Children's Hospital, Capital Medical University, National Center for Children's Health, Beijing 100045 China. ${ }^{3}$ Aegicare (Shenzhen) Technology Co., Ltd., Shenzhen 518110, China. ${ }^{4}$ School of Medicine, Jianghan University, Wuhan 430056, China. ${ }^{5}$ Radiology Department, Union Hospital, Huazhong University of Science and Technology, Wuhan 430056, China. ${ }^{6}$ Third People's Hospital of Hubei Province, Wuhan 430030, China. ${ }^{7}$ Center for Medical Genetics Department of Metabolism, Chiba Children's Hospital, Chiba 2660007, Japan. ${ }^{8}$ MILS International, Yokohama 2220033, Japan.

\section{Received: 11 October 2019 Accepted: 1 July 2020}

Published online: 16 July 2020

\section{References}

1. Peters H, Buck N, Wanders R, Ruiter J, Waterham H, Koster J, et al. ECHS mutations in Leigh disease: a new inborn error of metabolism affecting valine metabolism. Brain. 2014;137(11):2903-8.
2. Sharpe A, McKenzie M. Mitochondrial fatty acid oxidation disorders associated with short-chain enoyl-CoA hydratase (ECHS1) deficiency. Cells. 2018;7(6):46

3. Aulbert W, Weigt-Usinger K, Thiels C, Köhler C, Vorgerd M, Schreiner A, et al. Long survival in Leigh syndrome: new cases and review of literature. Neuropediatrics. 2014;45(06):346-53.

4. Fitzsimons PE, Alston CL, Bonnen PE, Hughes J, Crushell E, Geraghty MT, et al. Clinical, biochemical, and genetic features of four patients with shortchain enoyl-CoA hydratase (ECHS1) deficiency. Am J Med Genet A. 2018; 176(5):1115-27.

5. Ferdinandusse S, Waterham HR, Heales SJ, Brown GK, Hargreaves IP, Taanman J-W, et al. HIBCH mutations can cause Leigh-like disease with combined deficiency of multiple mitochondrial respiratory chain enzymes and pyruvate dehydrogenase. Orphanet J Rare Dis. 2013;8(1): 188.

6. Ferdinandusse S, Friederich MW, Burlina A, Ruiter JP, Coughlin CR, Dishop MK, et al. Clinical and biochemical characterization of four patients with mutations in ECHS1. Orphanet J Rare Dis. 2015;10(1):79.

7. Haack TB, Jackson CB, Murayama K, Kremer LS, Schaller A, Kotzaeridou $U$, et al. Deficiency of ECHS 1 causes mitochondrial encephalopathy with cardiac involvement. Ann Clin Transl Neurol. 2015;2(5):492-509.

8. Balasubramaniam S, Riley L, Bratkovic D, Ketteridge D, Manton N, Cowley M, et al. Unique presentation of cutis laxa with Leigh-like syndrome due to ECHS1 deficiency. J Inherit Metabolic Dis. 2017;40(5):745-7.

9. Tetreault M, Fahiminiya S, Antonicka H, Mitchell GA, Geraghty MT, Lines M, et al. Whole-exome sequencing identifies novel ECHS1 mutations in Leigh syndrome. Hum Genet. 2015;134(9):981-91.

10. Peters H, Ferdinandusse S, Ruiter JP, Wanders RJ, Boneh A, Pitt J. Metabolite studies in HIBCH and ECHS1 defects: implications for screening. Mol Genet Metab. 2015;115(4):168-73.

11. Frazier $A E$, Thorburn DR. Biochemical analyses of the electron transport chain complexes by spectrophotometry. Methods Mol Biol. 2012;837:49-62

12. Bernier F, Boneh A, Dennett X, Chow C, Cleary M, Thorburn D. Diagnostic criteria for respiratory chain disorders in adults and children. Neurology. 2002;59(9):1406-11.

13. Kremer $L S$, Prokisch $\mathrm{H}$. Identification of disease-causing mutations by functional complementation of patient-derived fibroblast cell lines. Methods Mol Biol. 2017;1567:391-406.

14. Ogawa E, Shimura M, Fushimi T, Tajika M, Ichimoto K, Matsunaga A, et al. Clinical validity of biochemical and molecular analysis in diagnosing Leigh syndrome: a study of 106 Japanese patients. J Inherit Metabolic Dis. 2017; 40(5):685-93.

15. Li H, Durbin R. Fast and accurate short read alignment with burrowswheeler transform. Bioinformatics. 2009;25(14):1754-60.

16. Wang K, Li M, Hakonarson H. ANNOVAR: functional annotation of genetic variants from high-throughput sequencing data. Nucleic Acids Res. 2010; 38(16):e164-e.

17. Alirezaie N, Kernohan KD, Hartley T, Majewski J, Hocking TD. ClinPred: prediction tool to identify disease-relevant nonsynonymous singlenucleotide variants. Am J Hum Genet. 2018;103(4):474-83.

18. Richards S, Aziz N, Bale S, Bick D, Das S, Gastier-Foster J, et al. Standards and guidelines for the interpretation of sequence variants: a joint consensus recommendation of the American College of Medical Genetics and Genomics and the Association for Molecular Pathology. Genet Med. 2015; 17(5):405.

19. Sakai C, Yamaguchi S, Sasaki M, Miyamoto Y, Matsushima Y, Yi G. ECHS1 mutations cause combined respiratory chain deficiency resulting in Leigh syndrome. Hum Mutat. 2015;36(2):232-9.

20. Li Q, Wang K. InterVar: clinical interpretation of genetic variants by the 2015 ACMG-AMP guidelines. Am J Hum Genet. 2017;100(2):267-80.

21. Carlston CM, Ferdinandusse S, Hobert JA, Mao R, Longo N. Extrapolation of variant phase in mitochondrial short-chain Enoyl-CoA hydratase (ECHS1) deficiency. JIMD Reports. 2018;43Springer:103-9.

22. Olgiati S, Skorvanek M, Quadri M, Minneboo M, Graafland J, Breedveld GJ, et al. Paroxysmal exercise-induced dystonia within the phenotypic spectrum of ECHS1 deficiency. Mov Disord. 2016;31(7):1041-8.

23. Al Mutairi F, Shamseldin HE, Alfadhel M, Rodenburg RJ, Alkuraya FS. A lethal neonatal phenotype of mitochondrial short-chain enoyl-CoA hydratase-1 deficiency. Clin Genet. 2017:91(4):629-33. 
24. Ganetzky RD, Bloom K, Ahrens-Nicklas R, Edmondson A, Deardorff MA, Bennett MJ, et al. ECHS1 deficiency as a cause of severe neonatal lactic acidosis. JIMD Reports. 2016;30:33-7.

\section{Publisher's Note}

Springer Nature remains neutral with regard to jurisdictional claims in published maps and institutional affiliations.

Ready to submit your research? Choose BMC and benefit from:

- fast, convenient online submission

- thorough peer review by experienced researchers in your field

- rapid publication on acceptance

- support for research data, including large and complex data types

- gold Open Access which fosters wider collaboration and increased citations

- maximum visibility for your research: over $100 \mathrm{M}$ website views per year

At BMC, research is always in progress. 